\title{
ENHANCED MRA IMAGES QUALITY USING STRUCTURE ADAPTIVE NOISE FILTER AND EDGE SHARPENING METHODS
}

\author{
Jawad Haider Kazmi ${ }^{1}$, Kalim Qureshi ${ }^{2}$, Haroon Rashid ${ }^{1}$ \\ ${ }^{1}$ Department of Computer Science, COMSATS Institute of Information Technology \\ University Road, Abbottabad ï 22060, Pakistan. \\ Email: \{jawadkazmi, haroon \}@ ciit.net.pk \\ ${ }^{2}$ Department of Math and Computer Science, \\ Kuwait University, Kuwait. \\ Email: qureshi@sci.kuniv.edu.kw
}

\begin{abstract}
MR imaging is an emerging and fast growing medical imaging technique which gives high quality images of the soft tissues. There are certain kinds of noise which contaminates these images and thus makes their interpretation difficult for both human and machine. Filtering is a mathematical technique in which intensities of each pixel of the input image are combined with the intensities of its neighboring pixels, to remove the noise and smooth the image. Filtering could be used with MR images for noise removal. The ordinary image filters blur the image and also remove important structural information like lines and edges. This loss of structural information could be dangerous in a clinical environment and could leads to incorrect diagnosis. To address this problem, a structure preserving noise filter is required. When images are processed for human vision, it is also desirable to make them pleasing by sharpening their edges. Such a structure adaptive noise (SAN) filter and an edge sharpening method is designed and implemented on our MRI Visualization toolkit. Our results show that the methods are effective in removing noise, preserving structure, and sharpening edges.
\end{abstract}

\section{Keywords: Medical imaging, MRA, adaptive image filters, edge sharpening}

\subsection{INTRODUCTION}

\subsection{Image Enhancement}

Image enhancement is a process principally focuses on processing an image in such a way that the processed image is more suitable than the original one for the specific application. The word ñspecificò has significance. It gives a clue that the results of such an operation are highly application dependent. In other words, an image enhancement technique that works well for X-ray topographic images may not work well for MR images.

The technique falls in two categories on the basis of the domain they are applied on. These are the frequency and spatial domains. The frequency domain methods works with the Fourier Transforms of the image. The term spatial domain refers to the whole of pixels of which an image is composed of. Spatial domain methods are procedures that operate directly on the pixels. The process can be expressed as:

$$
g(x, y)=T[f(x, y)]
$$

where $f(x, y)$ is the input image, $g(x, y)$ is the processed image, and $T$ is an operator on $f$ defined over some neighborhood of $(x, y)$ [1]. A number of enhancement techniques exist in the spatial domain. Among these are histogram processing, enhancement using arithmetic, and logical operations and filters.

\subsection{Medical Images}

Medical images are a special kind of images. These images are used for the diagnostics of diseases in the patients $[2,3]$. A number of modalities exist for obtaining these images. Among popular ones are Computed Topographic Imaging (CT), Magnetic Resonance Imaging (MRI), etc. Our focus here will be on the image obtained through Magnetic Resonance Imaging (MRI). 
Biologic tissues are comparatively transparent to x-rays and opaque to radiation with intermediate wavelengths when proceeding from the shorter to the longer wavelengths of the electromagnetic spectrum. This is true for ultraviolet, visible, and, to some extent, infrared light and microwaves. However, there is a window in tissue absorption through with radio waves can be used to probe deep inside the human body. The benefits derived from low-energy radiation and unprecedented level of information available from nuclear signals combined to make imaging by magnetic resonance a valuable biomedical imaging modality [4].

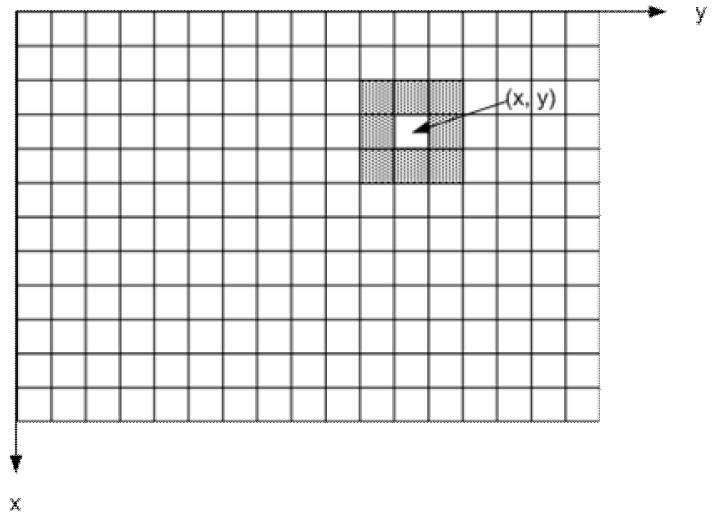

Fig. 1: The Spatial domain, the pixel (x, y) and the neighborhood of pixel (x,y)

\subsection{Magnetic resonance imaging (MRI)}

Magnetic resonance imaging (MRI) is a rapidly emerging technique that provides high quality images and potentially much more diagnostic information than conventional imaging modalities. MRI is conceptually quite different from currently used imaging methods. It is based on the principal of nuclear magnetic resonance imaging (NMR). The NMR principal, in brief, says that there are certain nuclei, in the nature that have an inherent spin property. The spinning of a charged nucleus results in a magnetic moment that behaves like a tiny bar magnet. The magnetic moment also spins or processes about an axis in a manner similar to the wobble of a spinning top. Hydrogen is the most frequently used nucleus for NMR imaging because of its abundance in biological tissue and its high NMR sensitivity.

This principal is depicted in Fig. 2. The complex nature of NMR allows a great deal of flexibility in image production and available information [5].

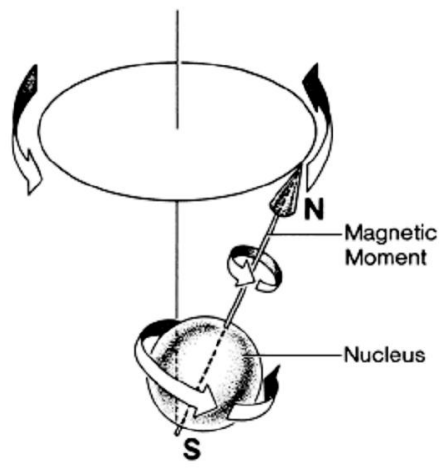

Fig. 2: The Spin property in the bases of NMR

\subsection{Magnetic Resonance Angiography (MRA)}

Magnetic resonance angiography (MRA) is the method of imaging blood flow in the brain. Fig. 3 [6] shows the different methods of obtaining the images of blood vessels. Since, MRA is based on the principal of NMR which 
dose not require the use of either ionizing radiation or the invasive application of contrast agents delivered through a catheter to produce an angiography, it is a safe method compared to other angiography techniques.

\subsection{Enhancement of MR Images}

Frequently MRI data is characterized by a relatively low signal to noise ratio (SNR) or contrast to noise ratio (CNR). The signal to noise ratio (SNR) is reversely proportional to the spatial resolution of the MR scan. Higher resolution means a noisier image. This is a tradeoff that the radiologist has to consider before each scan. When developing automated Computer Assisted Diagnostic (CAD) techniques, the errors introduced by the image noise are not acceptable. Thus, to limit these errors, a solution is to filter the data in order to increase the SNR. Filtering is a mathematical technique in which intensities of each pixel of the input image are combined with the intensities of its neighboring pixels, to remove the noise and smooth the image. Filters are also simple techniques for processing images. Filtering could be used with MR images for noise removal; the important property of the image filtering technique should be able to reduce the level of noise without the expense of feature preservation $[7,8]$.

The rest of the paper is organized as follows. In the next section we will discuss some related work. Section 3 and 4 discusses the design and workings of both the SAN filter and the edge sharpening methods. Section 5 presents the experiment details and results.

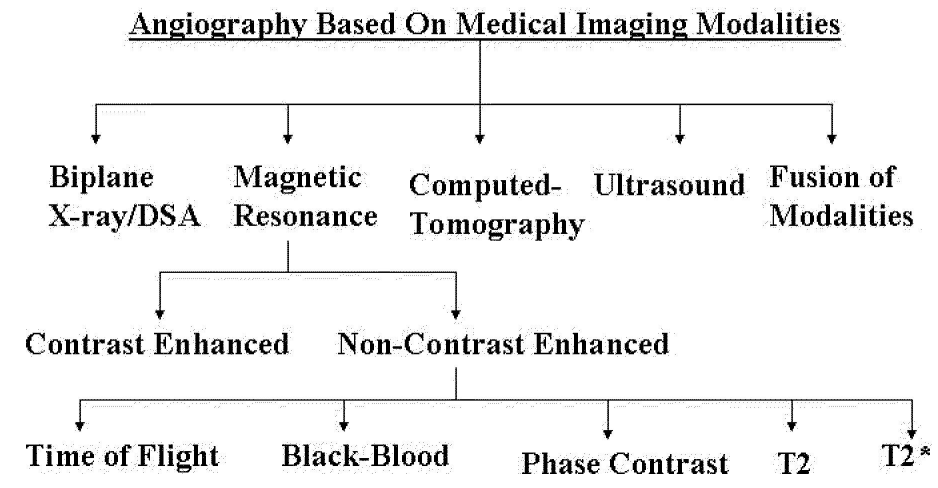

Fig. 3: Different methods of performing angiography [6]

\subsection{RELATED WORK}

Image smoothing techniques may be classified under two broad headings, linear and non-linear. Standard linear smoothing techniques based on local averaging or Gaussian weighted spatial operators reduce the level of noise, but this is achieved at the expense of poor feature preservation. Consequently, the filtered data appear blurry as step intensity discontinuities such as edges are attenuated. To compensate for these undesirable effects, non-linear techniques have been developed in order to achieve better feature preservation. Among these, the median filter is the simplest operator to remove impulse-like noise [9]. More complex non-linear techniques include statistical approaches based on non-parametric estimation $[10,11]$. However, while these methods do alleviate somewhat the shortcomings associated with linear techniques, they still perform only modestly when the data is affected by long tailed noise distributions. To complement these filtering approaches, a number of adaptive techniques have been proposed $[9,10,12]$. These methods try to achieve the best trade-offs between smoothing efficiency, feature preservation, and the generation of artifacts.

Another classification could be on the bases of frequency that the filtering method allows. High-pass and low-pass filters are in this category. It is well known that to reduce high-frequency random noise components in images, low-pass filters are necessary. Although noise filtering is essentially a smoothing process, simple low-pass filtering will blur image edges and other structures, damaging image fidelity [13]. These structures are significant to human viewers. They are also very important for automatic image analysis and synthesis, such as computer vision and image registration $[14,15]$. Some filtering algorithms have been proposed to address this problem successfully, such as the local statistics adaptive filtering algorithm [16], the gradient inverse weighted (GIW) filtering 
algorithm [17, 18], the multiple model Kalman filtering algorithm [19], and the directional filtering algorithm [11, 20]. Since, in medical diagnosis imaging, a study often requires a sequence of images, and digital images contain a large number of pixels, the processing efficiency of algorithms is another fundamental consideration in choosing algorithms for fast medical image processing.

As stated previously, low-pass filters are used for the reduction of high-frequency random noise in images. However, linear space invariant (LSI) low-pass filters blur the important image structures, such as edges and lines, and reduce the image contrast [21]. A solution in the low-pass domain is thus space variant (structure adaptive) filters. The quality of these filters is that they perform strong low-pass filtering only along the edges and lines and in the homogeneous (non-structured) areas of image.

Keeping all these things in mind; we have designed an adaptive structure preserving noise filter for medical images, which has the following qualities: (i) reduces the noise and preserves the image structure by minimizing the modifications to the original image, and (ii) provides a simple and fast implementation.

\subsection{THE DESIGN OF SAN FILTER}

The Fig. 4 shows the SAN Filter. Here, $x_{0}$ is the input image and $x_{l 0}$ is final filtered output image. Four low-pass image filters are defined. The function $f_{l i}(\cdot)$ represents the low-pass filter along the $i$ th direction and the index $i=$ $1,2,3,4$, correspond to $0^{\circ}, 45^{\circ}, 90^{\circ}, 135^{\circ}$, respectively. The low-pass filter generates four sub-images $x_{11}, x_{12}, x_{13}$ and $x_{14}$. Difference $d_{1}, d_{2}, d_{3}$ and $d_{4}$ are then calculated by subtracting each of the four sub-images from the original image. The output image $x_{l 0}$ is a synthesized image which is a weighted combination of the above four sub-images. In the diagram $\breve{E}_{i i}=w_{l i} \cdot x_{l i}$ is the output from each of the low-pass filter channel.

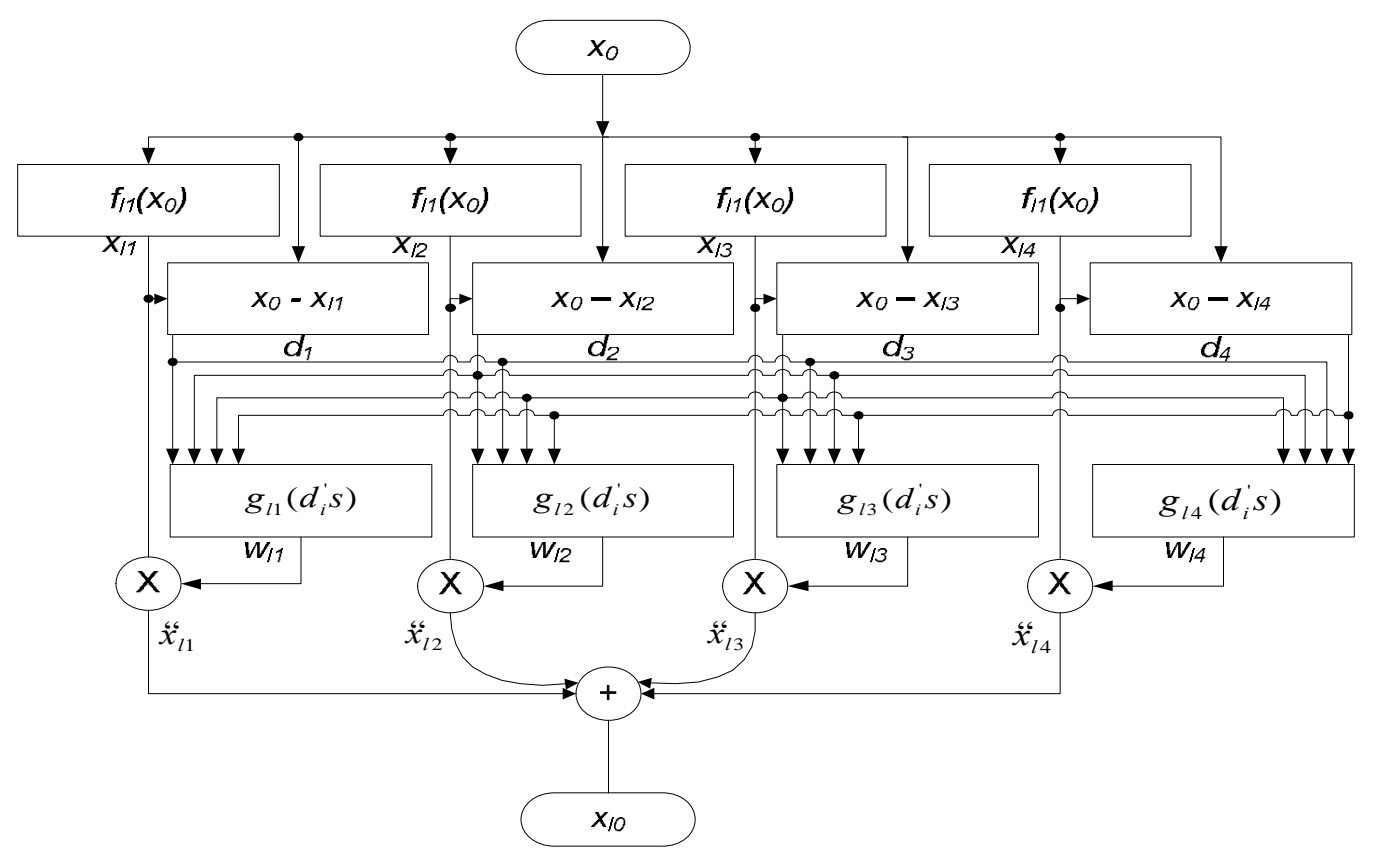

Fig. 4: The SAN filter

\subsection{Working of SAN Filter}

The SAN Filter tries to make minimum modification to the original image data, preserving the image structure by choosing the data from the outputs of four oriented low-pass filters while keeping certain filtering (smoothing) level in the homogeneous image areas. For example, if the pixel $x_{0}\left(n_{1}, n_{2}\right)$ is on an edge or a line along 0 degree, 
then the output $x_{l 1}\left(n_{1}, n_{2}\right)$ from the first filter $f_{l 1}\left(x_{0}\left(n_{1}, n_{2}\right)\right)$ will have much smaller difference from $x_{0}\left(n_{1}, n_{2}\right)$ at the position $\left(n_{1}, n_{2}\right)$ than the outputs from other three filters. In other words, $\bar{d}_{1}<\bar{d}_{i}$, for $i=2,3,4$.

Let $x_{0}\left(n_{1}, n_{2}\right)$ be the original image on a support $\mathrm{I}_{\mathrm{s}}$ four low-pass filters used to obtain four low-passed filtered sub-images along the four major directions (see Fig. 5).

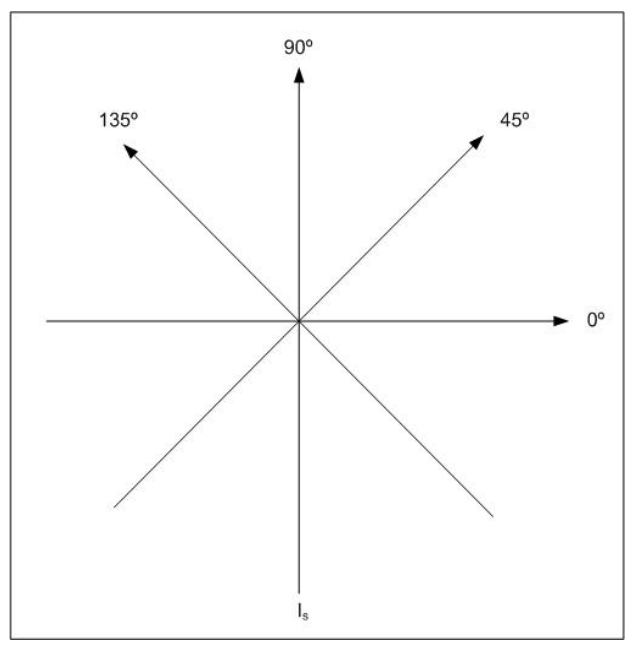

Fig. 5: The four major directions on which SAN filter is applied.

The four sub-images are defined as:

$$
\begin{aligned}
& x_{l 1}\left(n_{1}, n_{2}\right)=f_{l 1}\left(x_{0}\left(n_{1}, n_{2}\right)\right) ; \\
& x_{l 2}\left(n_{1}, n_{2}\right)=f_{l 2}\left(x_{0}\left(n_{1}, n_{2}\right)\right) ; \\
& x_{l 3}\left(n_{1}, n_{2}\right)=f_{l 3}\left(x_{0}\left(n_{1}, n_{2}\right)\right) ; \\
& x_{l 4}\left(n_{1}, n_{2}\right)=f_{l 4}\left(x_{0}\left(n_{1}, n_{2}\right)\right) .
\end{aligned}
$$

As previously mentioned, the function $f_{l i}(\cdot)$ represents the low-pass filter along the $i t h$ direction and the index $i=$ $1,2,3,4$, correspond to $0^{\circ}, 45^{\circ}, 90^{\circ}, 135^{\circ}$ respectively.

Taking a pixel and four of its neighborsôpixels around the position $\left(\mathrm{n}_{1}, \mathrm{n}_{2}\right)$ in the specified direction $\left(0^{\circ}, 45^{\circ}, 90^{\circ}\right.$, $135^{\circ}$ ) for low-pass filtering, the simple low-pass filter can be implemented in the image domain as:

$$
\begin{aligned}
& x_{l 1}\left(n_{1}, n_{2}\right)=\sum_{i=-2}^{+2} \alpha_{i} \cdot x_{0}\left(n_{1}+i, n_{2}\right) ; \\
& x_{l 2}\left(n_{1}, n_{2}\right)=\sum_{i=-2}^{+2} \alpha_{i} \cdot x_{0}\left(n_{1}+i, n_{2}+i\right) ; \\
& x_{l 3}\left(n_{1}, n_{2}\right)=\sum_{i=-2}^{+2} \alpha_{i} \cdot x_{0}\left(n_{1}, n_{2}+i\right) ; \\
& x_{l 4}\left(n_{1}, n_{2}\right)=\sum_{i=-2}^{+2} \alpha_{i} \cdot x_{0}\left(n_{1}+i, n_{2}-i\right) .
\end{aligned}
$$


The $\alpha_{i}$ is the weighting factor. Changing the value of this factor will change the characteristic of these filters. For this implementation we impose the following constrains on the value of $\alpha_{i}$

$$
\alpha_{+i}=\alpha_{-i} \text { and } \sum_{i=-2}^{+2} \alpha_{i}=1
$$

The implementation of the four filters for a central pixel and four neighboring pixels as defined in Equation 3 is depicted in Fig. 6. Here a white square represents an individual pixel of the image. The black square is the central pixel. The four neighbors along each direction for the central pixel are shown.

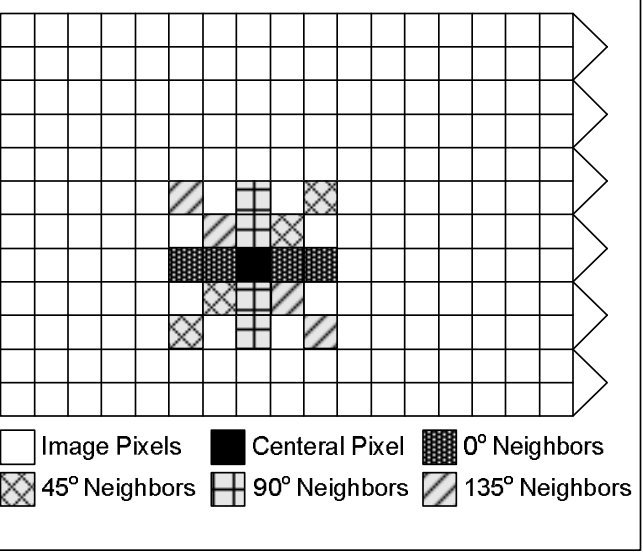

Fig. 6: The processing of SAN filter for single pixel

To calculate the difference $d_{1}, d_{2}, d_{3}$ and $d_{4}$, between the four sub-images $x_{11}, x_{12}, x_{13}$ and $x_{14}$ and the original image for a pixel $\left(n_{1}, n_{2}\right)$, the following equations are used:

$$
\begin{aligned}
& d_{1}\left(n_{1}, n_{2}\right)=x_{0}\left(n_{1}, n_{2}\right)-x_{l 1}\left(n_{1}, n_{2}\right) \\
& d_{2}\left(n_{1}, n_{2}\right)=x_{0}\left(n_{1}, n_{2}\right)-x_{l 2}\left(n_{1}, n_{2}\right) \\
& d_{3}\left(n_{1}, n_{2}\right)=x_{0}\left(n_{1}, n_{2}\right)-x_{l 3}\left(n_{1}, n_{2}\right) \\
& d_{4}\left(n_{1}, n_{2}\right)=x_{0}\left(n_{1}, n_{2}\right)-x_{l 4}\left(n_{1}, n_{2}\right)
\end{aligned}
$$

The absolute image difference of these images is defined as:

$$
\begin{aligned}
& \bar{d}_{1}\left(n_{1}, n_{2}\right)=\left|d_{1}\left(n_{1}, n_{2}\right)\right| ; \\
& \bar{d}_{2}\left(n_{1}, n_{2}\right)=\left|d_{2}\left(n_{1}, n_{2}\right)\right| ; \\
& \bar{d}_{3}\left(n_{1}, n_{2}\right)=\left|d_{3}\left(n_{1}, n_{2}\right)\right| ; \\
& \bar{d}_{4}\left(n_{1}, n_{2}\right)=\left|d_{4}\left(n_{1}, n_{2}\right)\right|
\end{aligned}
$$

The values of these differences are dependent on two factors. The first factor is the current operation location of the low-pass filter. When the low-pass filtering $f_{l i}\left(x_{l i}\left(n_{1}, n_{2}\right)\right)$ is along the edges or lines in certain image areas, these structures are smoothed and well preserved in the output $x_{l i}\left(n_{1}, n_{2}\right)$, and the resulting filtered image pixel value $x_{l i}\left(n_{1}, n_{2}\right)$ has a small difference $d_{i}\left(n_{1}, n_{2}\right)$ with the original image pixel value $x_{0}\left(n_{1}, n_{2}\right)$. On the other hand, when the low-pass filtering $f_{l i}\left(x_{l i}\left(n_{1}, n_{2}\right)\right)$ is across the edges or lines in certain image areas, these structures are severely distorted; therefore, the resulting image value $x_{l i}\left(n_{1}, n_{2}\right)$ has a big difference $d_{i}\left(n_{1}, n_{2}\right)$ with the original image pixel value $x_{0}\left(n_{1}, n_{2}\right)$. 
Second, the values of these differences are also dependent on the strengths of the low-pass filters. When stronger low-pass filters are applied, smoother images are produced, leading to big differences.

Since SAN Filter is a structure adaptive, the differences in the pixel values depend on the image structure. When the pixel $\left(n_{1}, n_{2}\right)$ belongs to an edge or lines, these structures are well preserved and smoothed in the output $x_{l i}\left(n_{1}\right.$, $\left.n_{2}\right)$. Hence, the resulting filtered image pixel value $x_{l i}\left(n_{1}, n_{2}\right)$ has a small difference $d i$ with the original image $x_{0}\left(n_{1}, n_{2}\right)$.

After discussing these preliminaries, we now start the calculation of the structured adaptive low-pass filter processed image $x_{l 0}\left(n_{1}, n_{2}\right)$ (our final output image):

$$
x_{l 0}=w_{l 1} \cdot x_{l 1}+w_{l 2} \cdot x_{l 2}+w_{l 3} \cdot x_{l 3}+w_{l 4} \cdot x_{l 4}
$$

where $w_{l 1}, w_{l 2}, w_{l 3}, w_{l 4}$ are the weighting parameters for the four low-pass filtered sub-images and are determined as some function $g_{l i}\left(d_{1}, d_{2}, d_{3} d_{4}\right)$ of the image differences.

The values of these weighting parameters should have conformance with:

$$
0 \leq w_{l i} \leq 1, \forall i=1,2,3,4
$$

For retaining the dynamic range of the original image $\mathrm{x}_{0}\left(\mathrm{n}_{1}, \mathrm{n}_{2}\right)$, these weighting parameters have the following relation.

$$
w_{l 1}\left(n_{1}, n_{2}\right)+w_{l 2}\left(n_{1}, n_{2}\right)+w_{l 3}\left(n_{1}, n_{2}\right)+w_{l 4}\left(n_{1}, n_{2}\right)=1, \forall\left(n_{1}, n_{2}\right)
$$

We have already discussed the relationship between the image structure and the differences $\left(d_{1}, d_{2}, d_{3}, d_{4}\right)$ calculated from the four low-passed filtered images $\left(x_{l 1}, x_{l 2}, x_{l 3}, x_{l 4}\right)$, To preserve the image structures, the subimages with smaller differences for the original image should have greater contribution to the final synthesized image. Therefore, the weighting parameters $w_{l i}(\mathrm{n} 1, \mathrm{n} 2)$ should be some monotonically decreasing function of the corresponding image difference $\bar{d}_{i}\left(n_{1}, n_{2}\right)$. One of the possibilities can be

$$
w_{l i}=\frac{s_{l}}{\bar{d}_{i}^{k}}
$$

where $s_{l}$ is a scaling factor for the constraint in Equation 9.

The weighting parameters can then be calculated from the image differences $\left(d_{1}, d_{2}, d_{3}, d_{4}\right)$ calculated above.

$$
\begin{aligned}
& w_{l i}=g_{l i}\left(d_{1}, d_{2}, d_{3}, d_{4}\right) \\
& g_{l i}\left(d_{1}, d_{2}, d_{3}, d_{4}\right)=\frac{\frac{1}{\bar{d}_{i}^{k}}}{\frac{1}{\bar{d}_{1}^{k}}+\frac{1}{\bar{d}_{2}^{k}}+\frac{1}{\bar{d}_{3}^{k}}+\frac{1}{\bar{d}_{4}^{k}}} \forall i=1,2,3,4 .
\end{aligned}
$$

Solving (7) and (11) we get

$$
x_{l 0}=\frac{\frac{1}{\bar{d}_{1}^{k}} \cdot x_{l 1}+\frac{1}{\bar{d}_{2}^{k}} \cdot x_{l 2}+\frac{1}{\bar{d}_{3}^{k}} \cdot x_{l 3}+\frac{1}{\bar{d}_{4}^{k}} \cdot x_{l 4}}{\frac{1}{\bar{d}_{1}^{k}}+\frac{1}{\bar{d}_{2}^{k}}+\frac{1}{\bar{d}_{3}^{k}}+\frac{1}{\bar{d}_{4}^{k}}}
$$

The filter in the Equation 12 is the SAN low-pass filter, which makes minimum modifications to the original image. In this way the structural information is preserved and the noise is filtered at the same time. The SAN filter 
achieves these by first generating four low-pass filtered sub-images and then uses the data from the output of these four sub-images.

If we take the value of power parameter $k$ in Equation 9 to 1, then, Equation 9 becomes

$$
x_{l 0}=\frac{\frac{1}{\bar{d}_{1}} \cdot x_{l 1}+\frac{1}{\bar{d}_{2}} \cdot x_{l 2}+\frac{1}{\bar{d}_{3}} \cdot x_{l 3}+\frac{1}{\bar{d}_{4}} \cdot x_{l 4}}{\frac{1}{\bar{d}_{1}}+\frac{1}{\bar{d}_{2}}+\frac{1}{\bar{d}_{3}}+\frac{1}{\bar{d}_{4}}}
$$

Equation 13 is the final low-pass SAN filter for original image $x_{0}\left(n_{1}, n_{2}\right)$ and the four sub-images $x_{l i}\left(n_{1}, n_{2}\right)$ for $i=1,2,3$ and 4 . The distances from the original image point to the four sub-image points are $\bar{d}_{1}, \bar{d}_{2}, \bar{d}_{3}$ and $\bar{d}_{4}$, respectively.

While $k \rightarrow+\infty$, we have

$$
x_{l 0}\left(n_{1}, n_{2}\right)=x_{l i}\left(n_{1}, n_{2}\right), \text { if } \bar{d}_{i}(n 1, n 2)<\bar{d}_{j}\left(n_{1}, n_{2}\right) .
$$

In this case, the final low-pass filtered image is determined as a nearest neighbor interpolation, and the filtering becomes a binary selection process from the outputs of the filter bank. It is noted that the filter in Equation 12 now becomes the directional averaging filter discussed in [13] for four directions. Although the filter defined by Equation 14 gives the minimum image difference by choosing only one from four given sub-images, the resulting smoothing is often not adequate in non-textured image areas. This is because only the output from the filter with the minimum $\bar{d}_{i}$ is used for low-pass filtering; only five pixels instead of seventeen pixels are utilized to smooth the noise even in homogeneous image areas. Increasing the number of pixels used in the filter in Equation 14 to achieve the same filtering strength as that given by Equation 12 in non-textured image areas will affect the sensitivity of the filter in Equation 14 to image structures. Thus, the filter structure in Equation 12 provides a better solution to the edge preserving noise reduction problem than the filter defined by Equation 14.

\subsection{DESIGN OF NON-SYMMETRIC EDGE SHARPENING SCHEME}

When images are processed for human viewers, it is often desirable to sharpen image edges for pleasing views. The most-used approach is the un-sharp masking. It is implemented by passing an image through a high-pass filter and then adding a fraction of its output to the original image [13], [15]. Although this approach yields reasonably good results in many cases, some undesired noise is still generated, especially when the original image has low signalto-noise ratio (SNR). 


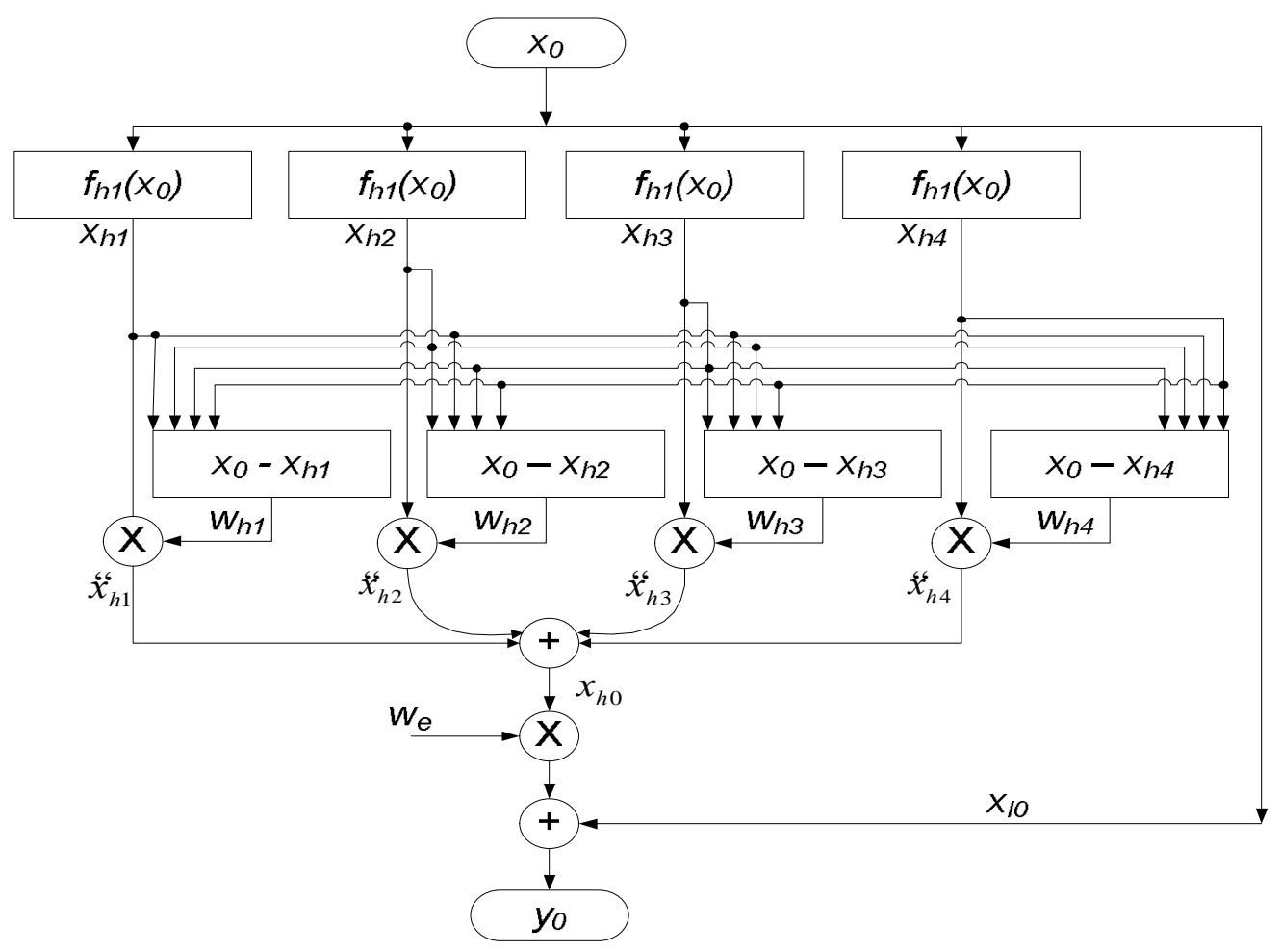

Fig. 7: Non-symmetric image edge sharpening scheme $\left(\breve{\mathscr{E}}_{h i}=w_{h i} \cdot x_{h i}\right)$

Fig. 7 shows a non-symmetric edge sharpening method to cope with this problem. The image data from the adaptive low-pass filter introduced in the previous section is high-pass filtered along the four major directions $\left(0^{\circ}\right.$, $45^{\circ}, 90^{\circ}$ and $\left.135^{\circ}\right)$ to obtain the outputs $x_{h 1}\left(n_{1}, n_{2}\right), x_{h 2}\left(n_{1}, n_{2}\right), x_{h 3}\left(n_{1}, n_{2}\right)$ and $x_{h 4}\left(n_{1}, n_{2}\right)$.

\subsection{Working of Edge Sharpening Scheme}

As in the case of SAN filter, let $x_{0}\left(n_{1}, n_{2}\right)$ the original image on the support $\mathrm{I}_{s}$, then the four sub-images along the four directions are calculated as:

$$
\begin{aligned}
& x_{h 1}\left(n_{1}, n_{2}\right)=f_{h 1}\left(x_{0}\left(n_{1}, n_{2}\right)\right) ; \\
& x_{h 2}\left(n_{1}, n_{2}\right)=f_{h 2}\left(x_{0}\left(n_{1}, n_{2}\right)\right) ; \\
& x_{h 3}\left(n_{1}, n_{2}\right)=f_{h 3}\left(x_{0}\left(n_{1}, n_{2}\right)\right) ; \\
& x_{h 4}\left(n_{1}, n_{2}\right)=f_{h 4}\left(x_{0}\left(n_{1}, n_{2}\right)\right) .
\end{aligned}
$$

Here, $f_{h i}(\cdot)$ for $i=1,2,3,4$ are I-D high-pass filters along the four major directions. The weighted summation of four high-pass sub-images is obtained as

$$
x_{h 0}=w_{h 1} \cdot x_{h 1}+w_{h 2} \cdot x_{h 2}+w_{h 3} \cdot x_{h 3}+w_{h 4} \cdot x_{h 4}
$$

The weighting parameters $w_{h i}$ are determined as functions of outputs of these high-pass filters and are constrained to have the relation

$$
w_{h 1}\left(n_{1}, n_{2}\right)+w_{h 2}\left(n_{1}, n_{2}\right)+w_{h 3}\left(n_{1}, n_{2}\right)+w_{h 4}\left(n_{1}, n_{2}\right)=1, \forall\left(n_{1}, n_{2}\right)
$$

To emphasize the high amplitude components from the cross edge direction, contrary to the low-pass filtering case, these weighting parameters should be some monotonously increasing functions of the amplitudes of the corresponding sub-images. A simple form of them is 


$$
w_{h i}=s_{h} \cdot\left|x_{h i}\right|^{j}
$$

where $s_{h}$ is a scaling factor for the constraint in Equation 17, and the superscript $j$ denotes the power parameter. For simple computations we assume that parameter $j$ is a positive integer. Using this definition and the constraint in Equation 14, these weighting parameters are determined by

$$
\begin{aligned}
& w_{h i}=g_{h i}\left(x_{h 1}, x_{h 2}, x_{h 3}, x_{h 4}\right) \\
& g_{h i}\left(x_{h 1}, x_{h 2}, x_{h 3}, x_{h 4}\right)=\frac{\left|x_{h i}\right|^{j}}{\left|x_{h 1}\right|^{j}+\left|x_{h 2}\right|^{j}+\left|x_{h 3}\right|^{j}+\left|x_{h 4}\right|^{j}} \forall i=1,2,3,4 .
\end{aligned}
$$

where the parameter $j$ can be used to adjust the performance of the filter.

Solving Equation 19 and 16, the high-pass filtered image data can be explicitly expressed as

$$
x_{h 0}=\frac{\left|x_{h 1}\right|^{j} \cdot x_{h 1}+\left|x_{h 2}\right|^{j} \cdot x_{h 2}+\left|x_{h 3}\right|^{j} \cdot x_{h 3}+\left|x_{h 4}\right|^{j} \cdot x_{h 4}}{\left|x_{h 1}\right|^{j}+\left|x_{h 2}\right|^{j}+\left|x_{h 3}\right|^{j}+\left|x_{h 4}\right|^{j}}
$$

This process chooses the high amplitude and high frequency components from filters in the directions across image structure edges. The filter outputs with higher amplitudes have bigger contributions to the final enhancement signal and a larger parameter $j$ makes this selection process more sensitive to the amplitudes of the high-pass filter outputs in different directions. Using this weighting process, image edges can be enhanced nonsymmetrical in four directions without much offsetting the low-pass filtering operation along edges in the previous section. As a special case, when $k \rightarrow+\infty$, only the output from the filter with the highest amplitude is activated for the edge enhancement as

$$
x_{h 0}\left(n_{1}, n_{2}\right)=x_{h m}\left(n_{1}, n_{2}\right), \text { if }\left|x_{h m}\left(n_{1}, n_{2}\right)\right|>\left|x_{h n}\left(n_{1}, n_{2}\right)\right|
$$

The high-pass filtered image for edge enhancement can be calculated as

$$
x_{h 0}=\frac{\left|d_{1}\right|^{j} \cdot d_{1}+\left|d_{2}\right|^{j} \cdot d_{2}+\left|d_{3}\right|^{j} \cdot d_{3}+\left|d_{4}\right|^{j} \cdot d_{4}}{\left|d_{1}\right|^{j}+\left|d_{2}\right|^{j}+\left|d_{3}\right|^{j}+\left|d_{4}\right|^{j}}
$$

Thus, the computation involved in the high-pass filtering is eliminated. Note that now the high-pass image $x_{h 0}$ cannot be independently adjusted since $d_{i}$ are determined by the specification of the low-pass filters in Equation 2 .

Finally, a fraction of these high-pass processed images $x_{h 0}\left(n_{1}, n_{2}\right)$ can be added to the low-pass processed image $x_{l 0}\left(n_{1}, n_{2}\right)$ to obtain the reduced final noise and edge-enhanced image $y_{0}\left(n_{1}, n_{2}\right)$, that is,

$$
y_{0}\left(n_{1}, n_{2}\right)=x_{l 0}\left(n_{1}, n_{2}\right)+w_{e} \cdot x_{h 0}\left(n_{1}, n_{2}\right)
$$

where $w_{e}$ is the edge enhancement weighting factor. This concludes our discussion on the working of SAN Filter and the edge sharpening method.

\subsection{RESULTS AND DISCUSSION}

We now discuss and evaluate the performance of the structure adaptive low-pass filter and the non-symmetric image edges sharpening scheme, in noise reduction, structure preserving, and processing speed. Several MRA volume set of varying resolutions are processed with both the filter and the edge sharpening scheme.

Before performing any experiments, the optimal values for the power parameters $k$ and $j$ (see Equations 11 to 19 in the discussion of the filter and edge sharpening method), and weighting parameters $w_{l 1}, w_{l 2}, w_{l 3}, w_{l 4}, w_{h 1}, w_{h 2}, w_{h 3}, w_{h 4}$ and $\alpha_{1}, \alpha_{2}, \alpha_{3}, \alpha_{4}$ must be known. 
There are a number of tradeoffs while selecting these values of power parameters $k$ and $j$. The first tradeoff is between noise reduction and edge preservation. Tuning for increased noise reduction will decrease the performance of the edge sharpening method. Increasing the value of $k$ will then decrease the performance of the edge sharpening method. The second tradeoff is between the better output and the computational power required for the processing. Although taking large value for $k$ and $j$ will make the filter more sensitive to image structures, the algorithm will become more computational intensive. We have done some experiments for the selection the values for these parameters, and have concluded that a value of $k$ between 3 and 5 and a value of $j$ between 2 and 4 will give a balanced result, keeping in view with the above mentioned tradeoffs.

$$
\begin{aligned}
& 3 \leq k \leq 5 \\
& 2 \leq j \leq 4
\end{aligned}
$$

We have chosen the value of 4 for $k$ and 3 for $j$. These values of are then used for the calculation of weighting parameters $w_{l 1}, w_{l 2}, w_{l 3}, w_{l 4}, w_{h 1}, w_{h 2}, w_{h 3}, w_{h 4}$. The values for $\alpha_{1}, \alpha_{2}, \alpha_{3}, \alpha_{4}$ are assumed to be:

$$
\alpha_{i}=\frac{1}{5}, \forall i=1,2,3,4 \text {. }
$$

The values of $w_{l 1}, w_{l 2}, w_{l 3}, w_{l 4}$ and $w_{h 1}, w_{h 2}, w_{h 3}, w_{h 4}$ are input dependent and therefore, cannot be specified here. These values will have to be calculated for each of the pixel of the input image using Equations 11 to 19.

The images selected for this experiment all belong to the same patient and have been obtained with different resolutions. In all of these images, the gray scale was quantized into 12 bits, which allowed 4096 different pixel intensities. Table 1 shows the dimension of these data sets. The data was in the raw image format. The visualization of these images was generated through the MRI Visualization toolkit which was developed as part of this project.

Table 1: Data sets used in this experiment

\begin{tabular}{ll}
\hline Type & Dimensions \\
\hline Data Set 1 & $512 \times 64 \times 512$ \\
Data Set 2 & $256 \times 128 \times 128$ \\
Data Set 3 & $256 \times 256 \times 128$ \\
\hline
\end{tabular}

The following three figures (Fig. 8, Fig. 9 and Fig. 10) show the MRA data before applying any of the SAN filter or edge sharpening method. Fig. 8 shows the three views of unprocessed data from the Data Set 1. Fig. 9 shows the visualization of Data Set 2, and Fig. 10 shows the Data Set 3ôs visualization.

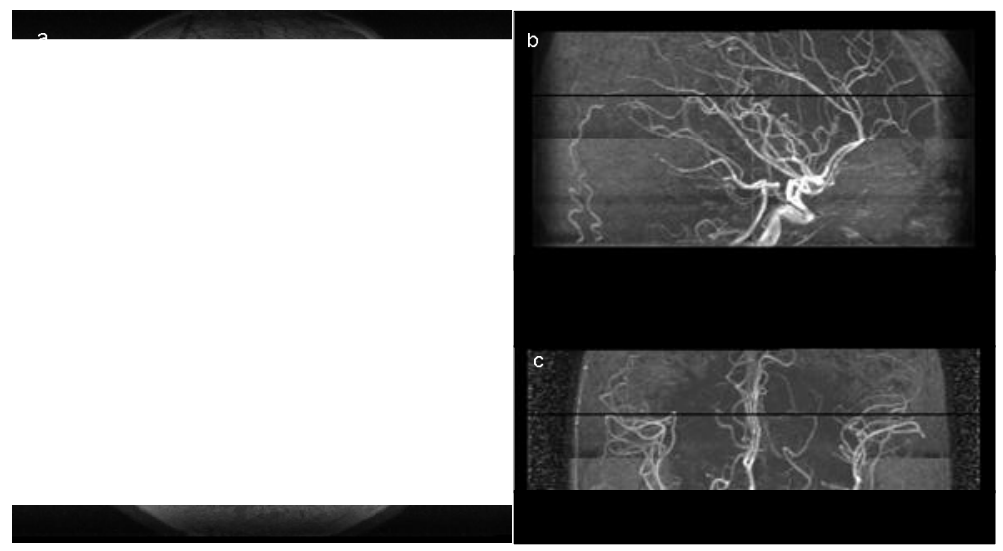

Fig. 8: Three views of Data Set $1(512 \times 64 \times 512)$ MRA data without SAN Filter and Edge Sharpening method 


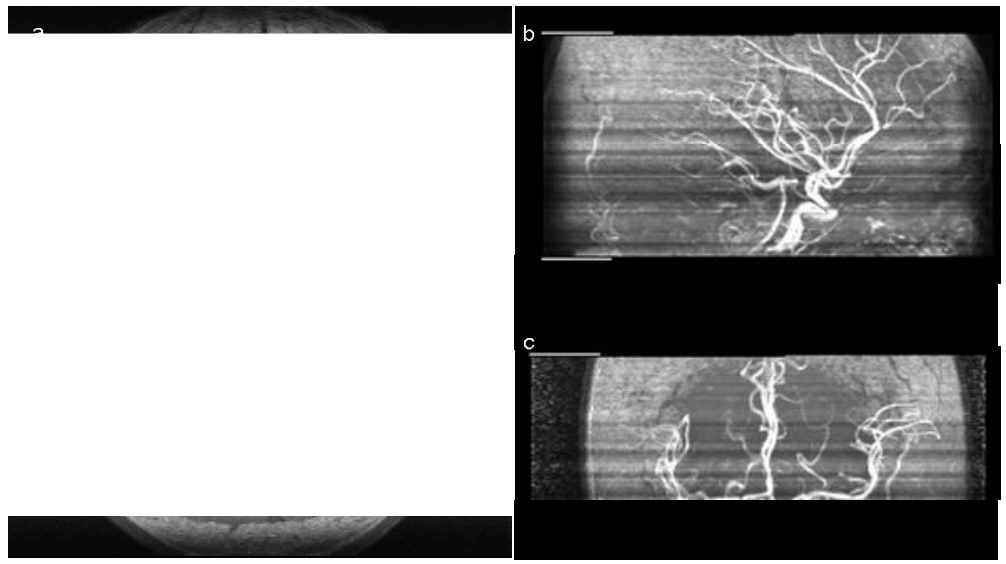

Fig. 9: Three views of Data Set 2 (256x128x128) MRA data without SAN Filter and Edge Sharpening method

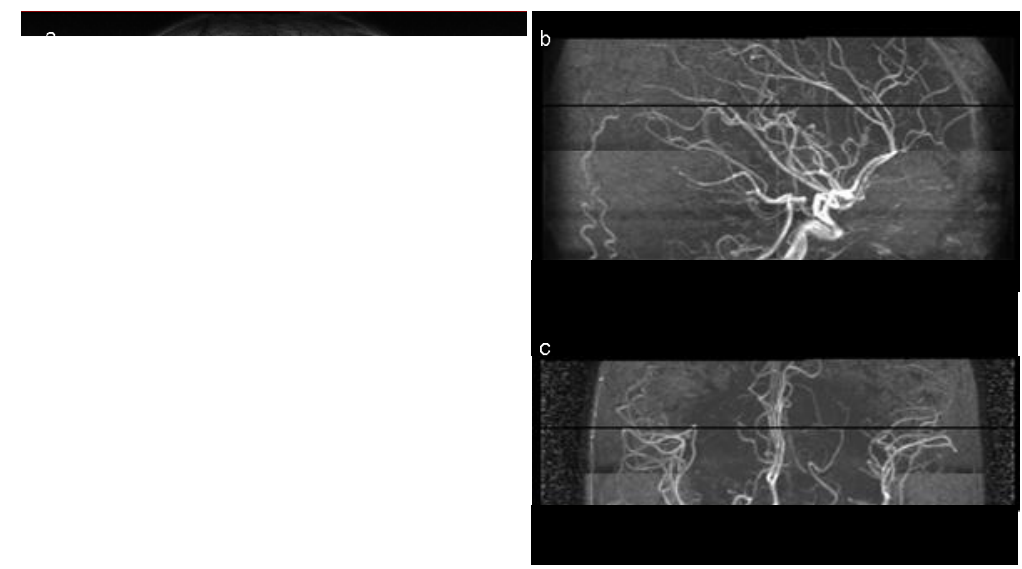

Fig. 10: Three views of Data Set 3 (256x256x128) MRA data without SAN Filter and Edge Sharpening method

After applying the filter on these three set of data, we have gathered the results, shown in the following three figures, (Fig. 11, Fig. 12, and Fig. 13).

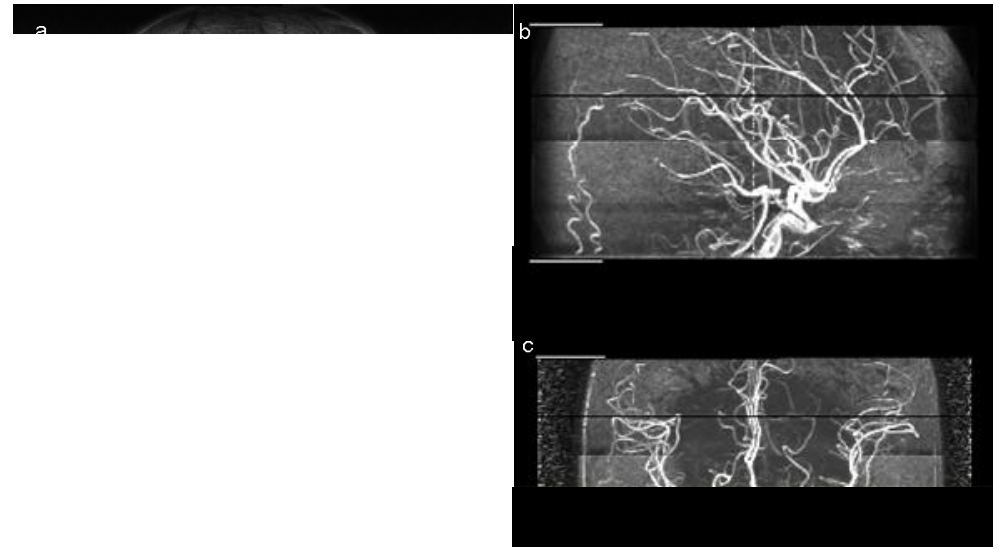

Fig. 11: Three views of filtered Data Set $1(512 \times 64 \times 512)$ MRA data showing the effects of applying SAN Filter and Edge sharpening method 


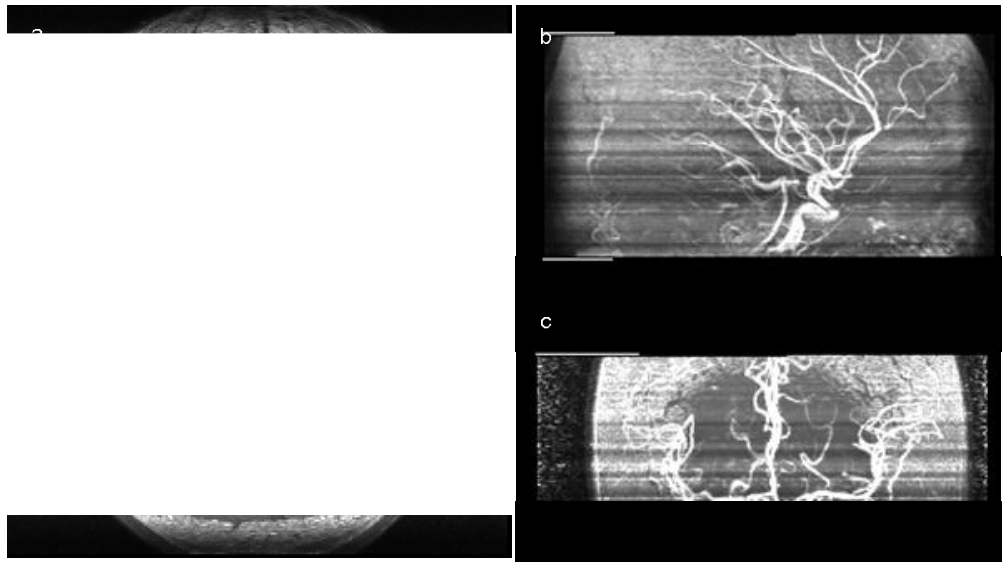

Fig. 12: Three views of filtered Data Set 2 (256x128x128) MRA data showing the effects of applying SAN Filter and Edge sharpening method
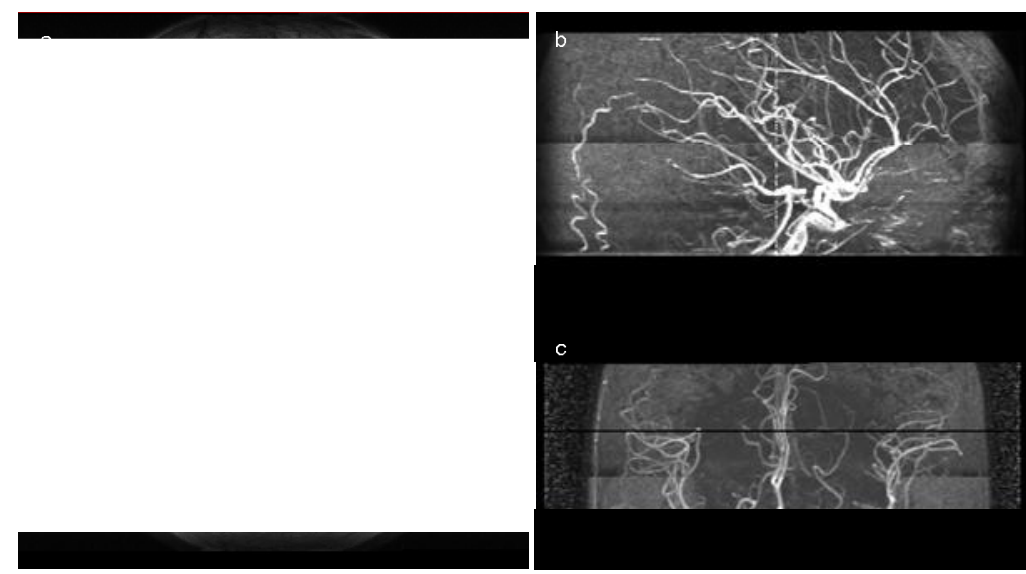

Fig. 13: Three views of filtered Data Set 3 (256x256x128) MRA data showing the effects of applying SAN Filter and Edge sharpening method

The running time of an algorithm is also an important measure of its performance. Table 2 shows the running times of the filter and edge sharpening method on the three sets of data stated above. The running time quoted here is the average of the 5 runs on each of the data sets, (the time taken by the MRI Visualization toolkit is not included in this time).

Table 2: Running times of the adaptive structured filter and edge sharpening method

\begin{tabular}{llll}
\hline Type & Dimensions & Size & Running Time \\
\hline Data Set 1 & $512 \times 64 \times 512$ & $32768 \mathrm{~kb}$ & $170.56 \mathrm{sec}$ \\
Data Set 2 & $256 \times 128 \times 128$ & $8192 \mathrm{~kb}$ & $103.00 \mathrm{sec}$ \\
Data Set 3 & $256 \times 256 \times 128$ & $16384 \mathrm{~kb}$ & $138.12 \mathrm{sec}$ \\
\hline
\end{tabular}

We have used Red Hat Fedora Core 6 with Kernel version 2.6.18-1.2748 on an Intel PIV, with 256 megabytes of RAM and $40 \mathrm{~GB}$ of hard drive. We used GCC version 4.1.1 as our compiler. X11 is used for the graphically display and visualizations.

The following two figures present a comparison of mean filter with SAN filter when applied to data set 1. 

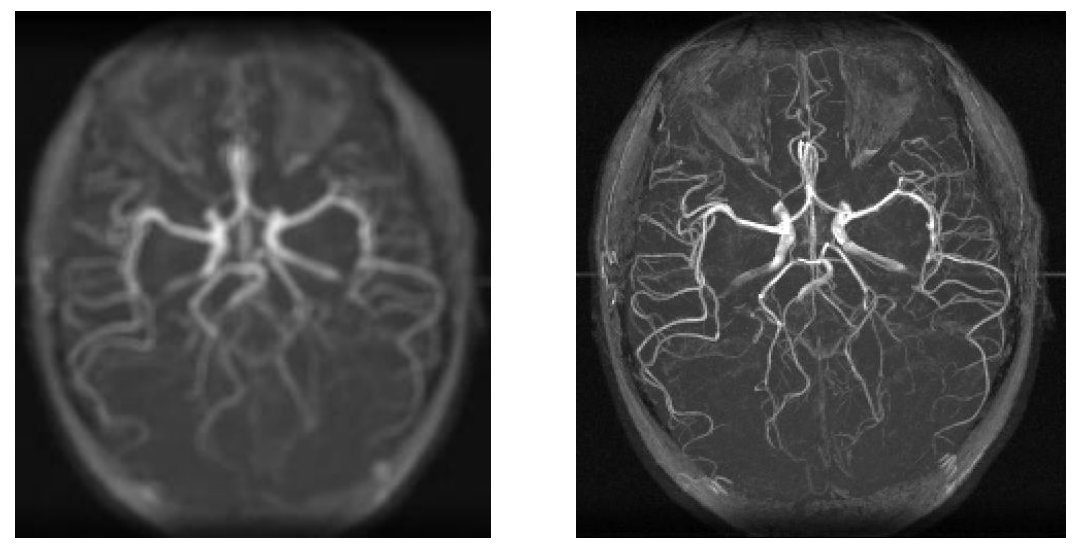

The next is a comparison between SAN filter and SAN Filter and Edge sharpening method when applied to data set 1 .
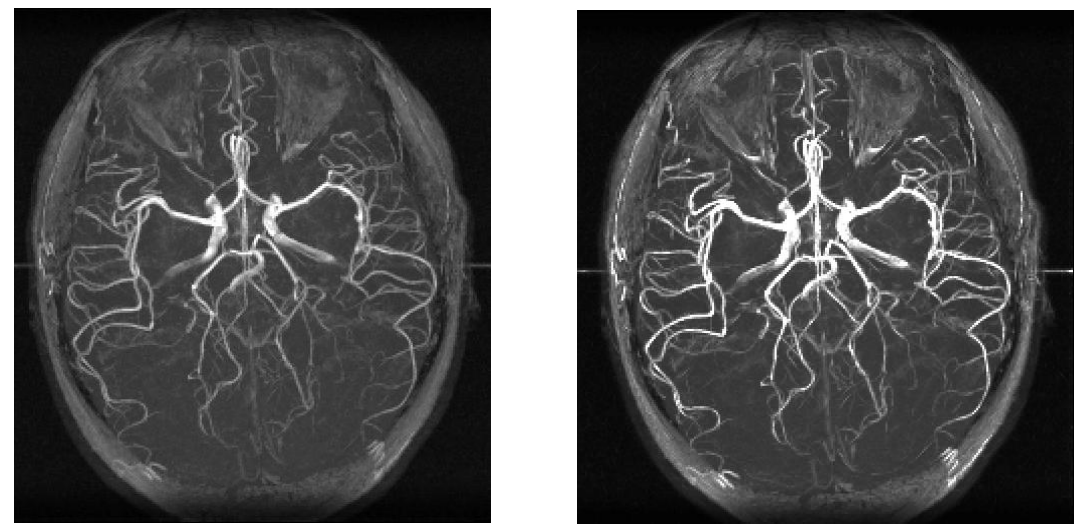

The last is a comparison between the mean filter and SAN filter and edge sharpening method.

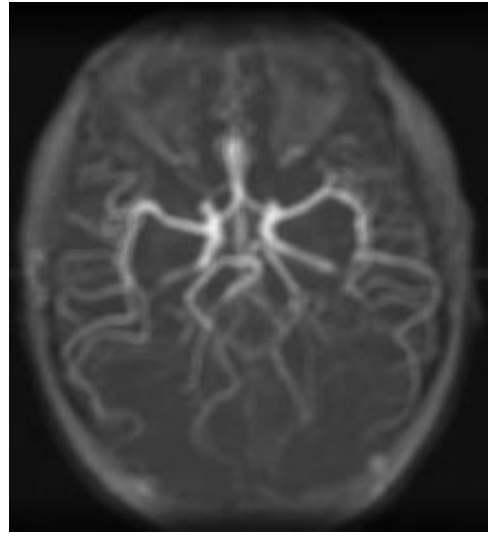

Fig. 14: Mean filter

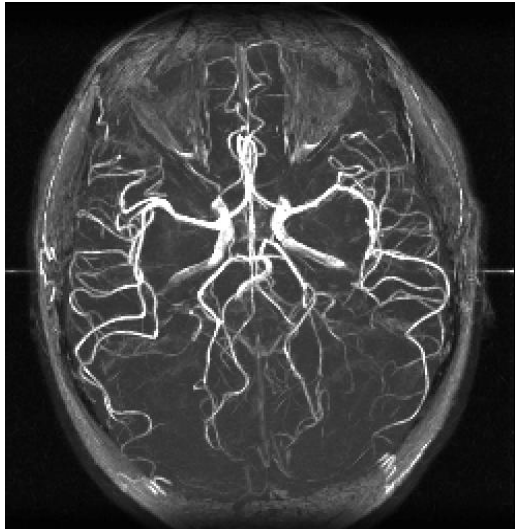

Fig. 15: SAN Filter and Edge Sharpening

\subsection{CONCLUSIONS AND FUTURE WORK}

In this research work, we have developed an MR image visualization tool as our test bed for accessing the effectiveness of different image enhancement and segmentation techniques. This tool can be utilized for such 
applications. We have experimented three different data set of MR angiography and MRI data of varying resolution, and have obtained good visualization in three different planes (sagittal, coronal and axial) in considerably optimal time. Due to these factors, the tool provides a valuable aid to any research with MR images. The other contribution in this work is the implementation of an adaptive structure preserving noise reduction filtering and non-symmetric edge sharpening method for MR images. Our results show that the method is effective in reducing noise, preserving structure, and sharpening edge. As a future work, we plan to develop a number of different applications on this test bed including automatic blood vessel extraction, registration of MR images, and incorporation of intelligence in the reduction the noise.

\section{REFERENCES}

[1] Gonzalez, R. C. and Woods, R. E. 2001 Digital Image Processing. (2 ${ }^{\text {nd }}$ ed). Addison-Wesley Longman Publishing Co., Inc.

[2] He, Huiguang; Tian, Jie; Zhao, Mingchang; Xue, Jian; Lu, Ke, ñ3D Medical Imaging Computation and Analysis Platformò, IEEE International Conference on Industrial Technology ICIT, Vol., Iss., 15-17, pp. 1160-1165, Dec. 2006.

[3] Latha Parthiban; R. Subramanian, ñMedical Image Denoising using X-letsò, Annual India Conference, Vol., Iss., pp. 1-6, Sept. 2006

[4] D.Stark and W.Bradley Jr., Ed. Magnetic Resonance Imaging, St. Louis, MO: Mosby, 1992.

[5] Steven E. Harms, Tommie J. Morgan, William S. Yamanashi, Thomas S. Harle, and Gerald D. Dodd, "Principles of nuclear magnetic resonance imaging", RadioGraphics, Vol. 4, pp. 26-43, 1984.

[6] J. S. Suri, K. Liu, L. Reden, and S. Laxminarayan, ñA review on MR vascular image processing algorithms: Acquisition and prefiltering: Part I,ò IEEE Trans. Inform. Technol. Biomed., vol. 6, pp. 324-337, Dec. 2002.

[7] Ovidiu Ghita, Kevin Robinson, Michael Lynch and Paul F. Whelan "MRI diffusion-based filtering: a note on performance characterisation", Vision Systems Group, School of Electronic Engineering, Dublin City University, Glasnevin, Dublin 9, Ireland, 2005.

[8] Yogo, Satoshi; Kobashi, Syoji; Kondo, Katsuya; Hata, Yutaka, ñ3-D Visualization of Rotator Cuff by Combining T1* and T2* MR Imagesò, World Automation Congress, WAC, Vol., Iss., 24-26, pp. 1-6, July 2006.

[9] M. Sonka, V. Hlavac and R. Boyle, Image processing, analysis and machine vision (2nd ed.), PWS, Boston, 1998.

[10] B. Smolka, R. Lukac, A. Chydzinski, K.N. Plataniotis and K. Wojciechovski, ñFast adaptive similarity based impulse noise reduction filter, Real Time Imagingò, Vol. 4, pp. 261ї 276, 2003.

[11] Kaijun Tang; Astola, J.; Neuvo, Y., "Nonlinear multivariate image filtering techniques", IEEE Transactions on Image Processing, Vol.4, Iss.6, pp. 788-798, Jun 1995.

[12] Comaniciu, D.; Meer, P., "Mean shift: a robust approach toward feature space analysis", IEEE Transactions on Pattern Analysis and Machine Intelligence, Vol.24, Iss.5, Pp. 603-619, May 2002.

[13] J. S. Lee, ñDigital image enhancement and noise filtering by use of local statistics,ò IEEE Transactions on Pattern Analysis and Machine Intelligence, Vol. PAMI-2, no. 2, pp. 165-168, March 1980.

[14] D. C. C. Wang, A. H. Vagucci, and C. C. Li, ñGradient inverse weighted scheme and the evaluation of its performance,ò Comput. Graph. Image Processing, Vol. 15, pp. 167-181, 1981. 
[15] X. Wang, ñOn the gradient inverse weighted filter,ò IEEE Transactions on Signal Processing, Vol. 40, no. 2, pp. 482-484, Feb. 1992.

[16] J. W. Woods, S. Dravida, and R. Mediavilla, ñmage estimation using doubly stochastic Gaussian random field models,ò IEEE Trans. Putt. pp. 165-168, Mar. 1980. Anal. Mach. Intell., vol. PAMI-9, pp. 245-253, Mar. 1987.

[17] J. S. Lim, Two-Dimensional Signal and Image Processing, Englewood Cliffs, NJ: Prentice Hall, 1990.

[18] L. B. Jackson, Digital Filters and Signal Processing. Kluwer Academic Publishers, 1989.

[19] L. L. Scharf, Statistical Signal Processing: Detection, Estimation and Time Series Analysis Reading, MA: Addison-Wesley Publishing Company, 1990

[20] L. Kaufman, D. M. Kramer, L. E. Crooks, and D. A. Ortendahl, ñMeasuring signal-to-noise ratios in MR imaging,ò Radiology 1989, vol. 173, pp. 265-267.

[21] Haiguang Chen; Li, A.; Kaufman, L.; Hale, J., ñA fast filtering algorithm for image enhancementò, IEEE Transactions on Medical Imaging, Vol.13, Iss.3, pp. 557-564, Sep 1994

\section{BIOGRAPHY}

Jawad Haider Kazmi is a graduate student and a Research Associate at Department of Computer Science at COMSATS Institute of Information Technology, Abbottabad Campus, Pakistan. He has more than 10 years of software design/development experience. He is currently working with Advanced Engineering Research Organization, Pakistan. His research interests includes medical image computing, parallel computing, distributed systems, computer networks, grid computing. His correspondence email address is jawadkazmi@ciit.net.pk.

Kalim Qureshi is a faulty member of Math and Computer Science Dept. Kuwait University, Kuwait and he is an adjunct Professor of Computer Science Dept. of COMSAT Institute of Information Technology, ABBOTTBAD, Pakistan. He is an approved su pervisor for MS/Ph.D thesis by High Education Commission, Islamabad, Pakistan. He published more than 30 International journal papers. His research interests include network parallel distributed computing; thread programming, concurrent algorithm designing, task scheduling, and performance measurement. Dr. Qureshi is a member of IEE Japan and IEEE Computer Society. His E-mail addresses are kalim@ciit.net.pk and qureshi@ @sci.kuniv.edu.kw.

Haroon Rashid is the Director and Faculty member of Department of Computer Science at COMSATS Institute of Information Technology, Abbattabad Campus, Pakistan. His research interests include parallel computing, distributed systems, high speed networks, multimedia, and network performance optimization. Dr. Haroon published numbers of journal/ conference papers. His E-mail address is haroon @ ciit.net.pk. 